criterion which does seem to call for the over-under arrangement, given the fact that the animal is more likely to change its orientation in the horizontal plane than in any other. It might be thought that a pair of electrodes in each of the three planes along with a scrambler to shift the voltage rapidly from pair to pair would provide a general. solution to the problem, but that is not so. With such an arrangement, the electrodes inactive at any given moment (being better conductors than water) would carry most of the current.

\section{REFERENCES}

HOLMES, N. K., \& BITTERMAN, M. E. Measurement of consummatory behavior in the fish. Journal of the Experimental Analysis of Behavior, 1969, 12, 39-41.

HORNER J L. LONGO N. \& BITTERMAN, M. E. A shuttle box for the fish and a control circuit of general applicability. American Journal of
Psychology, 1961, 74, 114-120

KELLOGG, W. N. Gajvanotropism as an avoidance response. Journal of Comparative \& Physiological Psychology. $1958,51,652-657$.

LONGO, N., HOLLAND, L. R., \& BITTERMAN, M. E. The resistive sheet: A gridless and wireless shocking technique, American Journal of Psychology, 1961, 74, 61 2-618.

WILSON, W. L., DARCY, J, M. \& H A RALSON, J. V. Reserpine and conditioned suppression in the fish Tilapia h. macrocephala. Psychonomic Science, 1970, 20, 47-49.

\title{
A telemetry system for measuring chewing behavior in humans*
}

\author{
JOHN D. RUGH \\ University of California at Santa Barbara, Santa Barbara, California 93016
}

A system is described for detecting and recording human chewing behavior. A small transmitter mounted in the side frame of eyeglasses was used to detect muscle movement associated with chewing. The transmitter signal was received and converted to a direct-current voltage which varied at a rate proportional to the S's chewing movement. Exploratory data are presented which suggest that individuals chew at about the same rate per second, but vary in the total number of chews emitted to consume the same amount of foodstuff.

Very little descriptive or experimental work has been done by psychologists on the chewing behavior of humans. With the exception of some early work by Hollingworth (1939) and Freeman (1940), dealing with the relationship between chewing and tension reduction, chewing has been of interest primarily to dentistry and nutrition.

To date, the methods employed for studying human chewing have a number of characteristics which make them undesirable for most psychological studies. Hollingworth (1939), for example, measured the response by visual observation, a method limited to gross measures, such as chewing or not chewing. Duckworth and Shirlaw (1958), recording chewing in cows, placed an inflated balloon between the animal's jaw and head strap. The jaw movements varied the balloon air pressure, which was recorded. Although precise measures such as chews per minute can be recorded using this method, it is limited to animals whose chewing involves large jaw movement and would be difficult to use with humans.

* This research was supported in part by United States Public Health Service Grant MH-15616 to David Premack. The author wishes to thank David Premack and Eric Jacobson for their criticism of the manuscript and George Stevens for manuscript and
Electromyographic (EMG) methods have been used extensively to study muscle activity associated with chewing movements (see Kawamura, 1964, for a review of the literature). Measuring EMG, however, requires that electrodes be attached to the S's skin over a muscle or under the skin with wires leading to the recording equipment. This limits the S's mobility and undoubtedly calls his attention to the behavior under study. And, of course, EMG recordings are frequently affected by the S's emotional state, head position, and the type of electrodes used (Kawamura, 1964).

Another method is the cinematographic technique (Beyron, 1964; Ahlgren, 1966). Films are made of the S's chewing and later analyzed, using a projector. A great deal of information can be gleaned from the films, but the method usually requires that the $S$ 's head remain stationary and that clamps be fitted such that the S's lips are held open while chewing.

Tooth transmitters have recently been developed (Kavanagh \& Zander, 1965; Roeber, Pameijer, \& Glickman, 1968) which send bite information to nearby receivers and recorders. Although an excellent method, the use of tooth transmitters is generally restricted to dentists who have the technical ability to install such devices. This procedure would also be difficult to employ without the S's being aware of the purpose of the experiment. It is not clear as yet what effect $S$ awareness may have on the S's chewing behavior. However, when a $S$ knows that chewing behavior is being observed, it is likely that he consciously controls the movements. These consciously controlled movements may be considerably different from the more normal nonobserved chewing patterns. The telemetry system described below overcomes the above limitations and provides a method of measuring and recording detailed chewing information.

\section{APPARATUS}

\section{General Description}

Chewing in humans involves the contraction and relaxation of the temporalis muscle near the temple area. When the jaw is clenched, the muscle contracts and produces a slight bulge on the surface of the skin near the temple. In our laboratory, this movement is used to frequency-modulate a small transmitter concealed in the side frame of a set of eyeglasses worn by the $S$. The transmitted signal is received (see Fig. 1) and mixed with the receiver's beat frequency oscillator (BFO) signal to generate an audio output. This audio output varies in frequency as the individual chews, i.e., it increases when the $S$ "bites down" and decreases when the jaws open.

With this technique the audio signal may be tape-recorded and analyzed at a later time, and/or it may be fed directly into a frequency-to-voltage converter circuit. The output of the frequency-to-voltage circuit is a dc voltage varying at a rate proportional to the chewing of the $\mathrm{S}$. The dc signal may be recorded on a chart recorder for later analysis or digitalized via an analogue-to-digital converter and analyzed on a small computer.

\section{Transmitter}

The transmitter (see Fig. 2) is a $3-\mathrm{MHz}$ oscillator circuit, similar to the one described by Wolff (1967). The components of the transmitter are mounted in holes cut in the side frame of a set of wide-framed eyeglasses. 


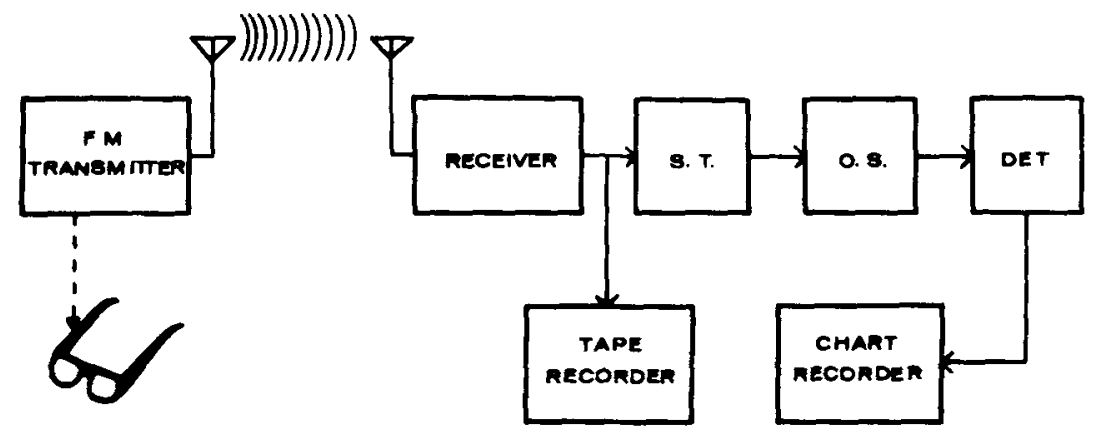

Fig. 1. Pictorial diagram of telemetry system. The Schmitt trigger (ST), one-shot (OS), and detector (Det) make up a frequency-to-voltage converter circuit.

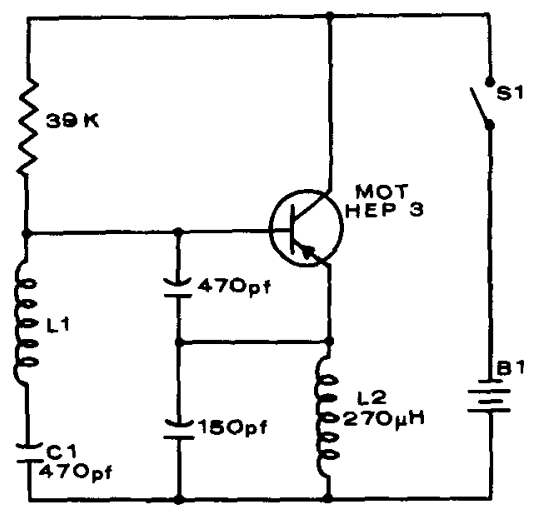

Fig. 2. Circuit diagram of transmitter. $B_{1}$ refers to two Eveready E-312 mercury cells. Coil $\mathrm{L}_{2}$ is a Millex subminiature choke, Part No. 70F274A1. Capacitor $\mathrm{C}_{1}$ and Coil $L_{1}$ determine the frequency of oscillation. Switch $S_{1}$ is a tilt-type mercury switch, Waber Electronics, Model 3040, positioned such that it is on when the glasses are right side up and off when turned upside down. Coil $L_{1}$ is 75 turns of No. 34 copper wire wound on a form with a $4-\mathrm{mm}$ center diameter and a 2-mm width. Ohmite $1 / 8 \cdot W$ resistors and Aerorox Corp. Type ULA chip capacitors were used because of their small size. Later models were built using a less temperature-sensitive silicon HEP-52 transistor made by Motorola and a small magnetic reed switch, Type MMRR-2, made by Hamlin, Inc.

Coil $\mathrm{L}_{1}$ is located such that it is less than $5 \mathrm{~mm}$ from the $S$ 's temple when the glasses are in position. If Coil $L_{1}$ is more than $5 \mathrm{~mm}$ from that position, a small piece of aluminum may be taped to the S's temple. This greatly increases the sensitivity of the system. An alternative method, which was found valuable, is to mount Coil $L_{1}$ on the end of a small screw, spring, and nut arrangement, such that the position of the coil may be adjusted allows the $\mathrm{E}$ to compensate for various head sizes.

Coil $L_{2}$ is separated as far as possible from Coil $L_{t}$ to prevent loading. All components and the holes they are placed in are covered with Palette plastic resin pigmented to match the original color of the glasses. The transmitter components and glasses cost approximately $\$ 15$. Battery life is about $40 \mathrm{~h}$.

Receiver and Frequency-to-Voltage Converter

A Hammarlund Model SP-600 short-wave receiver, purchased used for $\$ 300$, was used to receive the transmitted signal. A less expensive Knight $\mathrm{R}-55 \mathrm{~A}$ receiver $(\$ 60)$ was found to work equally well at close ranges. Of course, the maximum transmission range depends upon receiver sensitivity, selectivity, local interference, and antenna placement. In general, the range is between 5 and $10 \mathrm{ft}$. The frequency-to-voltage converter circuit, described elsewhere (Rugh, 1970), consisted of three commercially available digital logic packages. As indicated in Fig. 1, the output of the receiver was fed directly into a Schmitt trigger (BRS ST-102, BRS Electronics, Beltsville, Md. 20705), whose output was fed into a monostable multivibrator (BRS OS-101) set at 60 microsec. The output of the monostable multivibrator was fed through a 1-h choke. A 10-microF capacitor, connected from the choke output to ground, reduced the ripple voltage to less than $0.05 \mathrm{~V}$ (rms). The converter has a linear frequency-to-voltage function from $50 \mathrm{~Hz}$ to $11 \mathrm{kHz}$ and an output from $-11 \mathrm{~V}$ to $-2 \mathrm{~V} d c$, depending upon the input frequency. This output was fed directly into a chart recorder. with respect to the S's temple. This

The telemetry system described above is capable of detecting several aspects of human chewing behavior. These include the force and duration of each bite, the total bites emitted during a period of time, and chew cycle times. To demonstrate that the system does measure each of the above aspects and to determine the system's limitations, a series of tests were made. In general, the tests involved comparing the data recorded using the telemetry system with data recorded using more conventional techniques such as visual observation and recordings from a gnathodynamometer, an instrument for the measurement of biting force.

The four Ss ranged in age from 24 to 48 years. For testing, each $\mathrm{S}$ was seated at a small table in a quiet room which contained the equipment rack and was told that he was participating in a study designed to test the reliability of a new technique to study chewing behavior. A pair of glasses was selected to fit the individual's head snuggly, yet comfortably, and he was asked to chew a stick of gum for $3 \mathrm{~min}$. This $3-\mathrm{min}$ period was necessary to allow the transmitter in the glasses to warm up to the S's body temperature. Several of the system components were slightly temperature sensitive, and the oscillator's frequency drifted as the components warmed to body temperature. Later models of the glasses were built using components which were less temperature sensitive, so a warm-up period was unnecessary. During the 3-min period, the receiver was tuned to the transmitter's frequency, and the receiver's BFO was adjusted, such that a $1-\mathrm{kHz}$ tone could be heard in earphones plugged into the receiver. This tone was also monitored on a Hewlett-Packard frequency meter, Model 500A. With the S chewing, the distance between Coil $L_{1}$ and the skin surface was adjusted, such that his chewing movements produced a frequency shift of approximately $5 \mathrm{kHz}$. If the frequency variations caused by jaw movements were too great, Coil $\mathrm{L}_{1}$ was moved further from the S's skin surface. If the frequency variations were too small, Coil $\mathrm{L}_{1}$ was moved closer. Thus, in general, the sensitivity of the system is greater the closer the coil is to the muscle movement.

\section{Measure of Force}

Validity measures of the system's ability to detect the force and duration of each bite were made by asking Ss who wore the glasses to bite at different strengths and for varied durations on the sensitive element of a strain gauge gnathodynamometer. The 


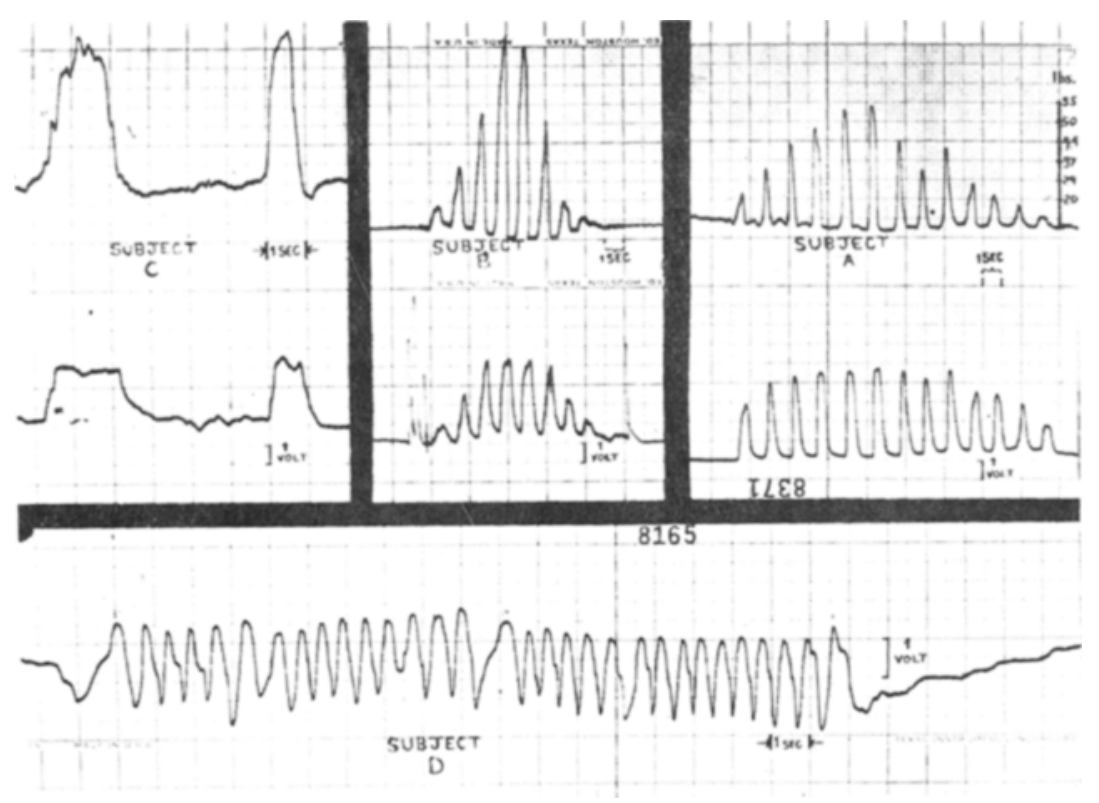

Fig. 3. Oscilloriter recordings of human chewing. The upper three plates are simultaneous recordings of the gnathodynamometer output (upper trace) and the output of the chew detector (lower trace). The lower plate is the chew detector recording of a $S$ eating one peanut. Each upswing in voltage is a "biting down" and each downswing is an opening of the jaws. The first chew emitted is on the right and the trace proceeds from right to left. Chews 16 and 31 are chews associated with swallowing.
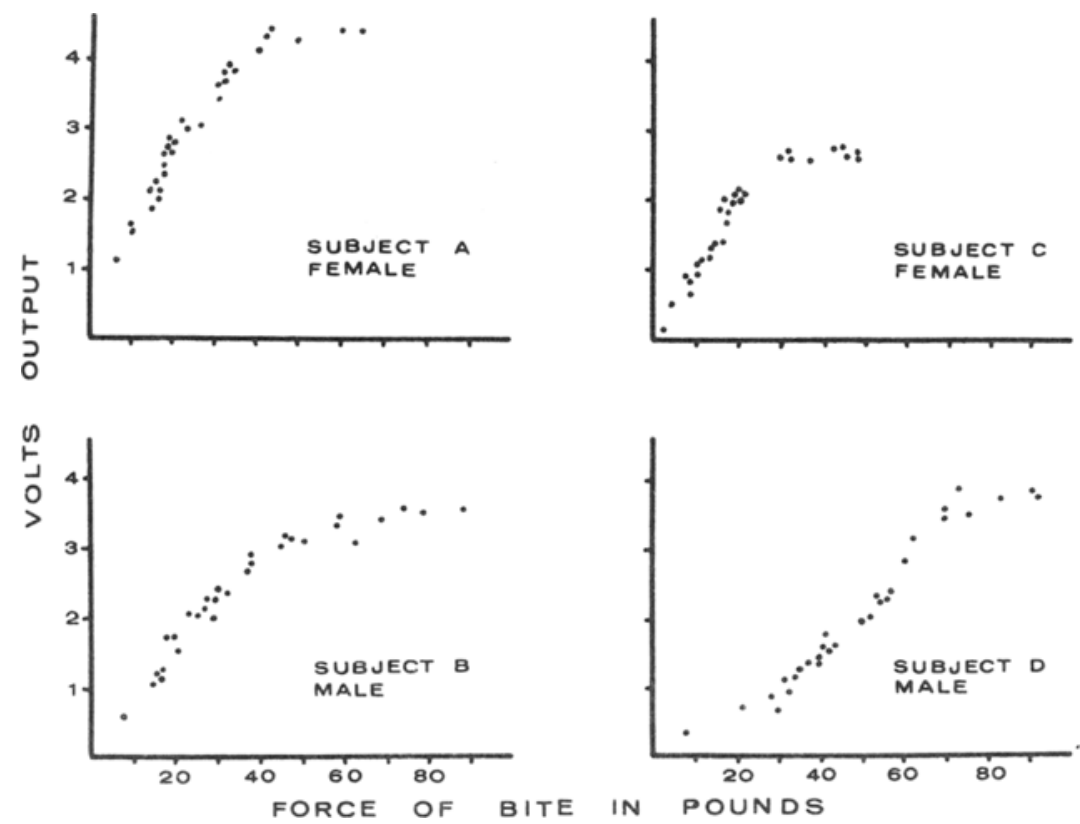

Fig. 4. Relationship of biting force, as measured by a gnathodynamometer and voltage output of the telemetry system.

chewing gum was removed, and the sensitive element of the gnathodynamometer was placed between the first molars on the right side of the mouth. The biting surface was approximately $2 \mathrm{sq} \mathrm{mm}$, and the thickness of the element was $3 \mathrm{~mm}$. The voltage outputs of the telemetry by the gnathodynamometer, the greater is the voltage output of the telemetry system; however, the relationship is not linear, nor is it the same for each $\mathrm{S}$. The relationship between biting force as measured by the gnathodynamometer and the voltage output of the telemetry system is shown for each $\mathrm{S}$ in Fig. 4. These points were taken from the last 30 bites made by each $S$ during the above-mentioned test. Note that at high magnitudes of biting force, increases in force no longer are accompanied by increases in voltage output of the chew detector; thus, at high biting forces the telemetry system is insensitive to different degrees of force. It should be noted, however, that the pressure used for ordinary chewing is, on the average, about one-third of the maximum force that can be exerted (Jenkins, 1960). The maximum biting force for males is about $119 \mathrm{lbs}$; for females, about $85 \mathrm{lbs}$ (Klatsky, 1942). The normal chewing force is thus approximately $39 \mathrm{lbs}$ for males and approximately $28 \mathrm{lbs}$ for females. Figure 4 illustrates that for these ranges of pressure the system does detect differences in biting force. The minimum biting force detectable varied from $S$ to $S$ but has always been found to be less than $1 \mathrm{lb}$. When absolute measures of biting forces are desired, a calibration plot similar to those of Fig. 4 must be made for each $\mathrm{S}$.

The amount of voltage change produced by a given biting force is dependent upon the placement of Coil $L_{1}$, i.e., its position with respect to the temporalis muscle; thus, any movement of the glasses from their original position will change the calibration and give incorrect absolute force values. Shifts in the position of the glasses are readily apparent, as the $1-\mathrm{kHz}$ tone in the earphones suddenly shifts and does not return to $1 \mathrm{kHz}$. When a shift does occur, the glasses can be readjusted to their original position. The correct original position can be identified by the recurrence of the $1 \cdot \mathrm{kHz}$ tone in the earphones. By ensuring that the glasses fit snuggly, shifting seldom occurs. Each $S$ in the present study was asked to walk, talk, and nod his head during a later test. These movements did not cause slippage; however, slippage did occur twice during another test. One resulted when a $\mathbf{S}$ sneezed rather violently, and the other slippage occurred when a $S$ rested his head in his hands, thus pushing the glasses from their original position. In both cases the $\mathrm{E}$ was able to readjust the glasses to their original position.

Absolute measures of biting force would, of course, be difficult to obtain without a S's being aware of the 
purpose, since the system's voltage output must be calibrated with the output of a gnathodynamometer. Relative force measures, however, do not require calibration and can thus be made without informing the $S$ of the behavior under study.

Measures of Duration of Bites

The recordings displayed in Fig. 3 (upper frames) attest to the system's ability to detect the duration of each bite and the chew cycle times. The voltage output of the telemetry system increases when the $S$ bites down on the gnathodynamometer and does not decrease until the biting force is removed. The duration of each bite is easily calculated by measuring the time elapsed between the rise and fall of the voltage. Chew cycle times are calculated by measuring the time elapsed between a rise in voltage and the following rise. No calibration is necessary for these measures, and slight slippage of the glasses does not adversely affect the recordings. Since no calibration is necessary, both measures may easily be made without informing the $S$ of the purpose.

\section{Measures of Total Chews Emitted}

A test of the system's ability to record accurately the number of chews emitted was made by asking each $\mathbf{S}$ to eat 10 peanuts, one at a time, and to count and record the number of chews emitted per peanut. The numbers reported were later compared with the number of chewing movements recorded using the telemetry system. An examination of the first records revealed that the S's count was less than the chew detector by two or three chews per peanut. It was found that this descrepancy was due to the chew detector's recording the biting movements which took place during swallowing, which the $\mathrm{S}$ had ignored. The remaining three Ss were instructed to record their swallows as well as their chews. The number of chews recorded by the telemetry system and the number reported by the $S$ were the same for 27 of the remaining 30 peanuts eaten. The discrepancies of one, two, and four chews per peanut may have been due to $S$ error in counting, as in each case the telemetry system recorded more chews than the $S$ reported. The average number of chews per peanut was 22.6, and the range was 12 to 32 chews. Figure 3 (lower frame) is an example of the data recorded.

After some experience in reading the recordings, the biting associated with swallowing can be distinguished from normal chewing, as the biting associated with swallowing is typically of longer duration than normal chewing. (Note the 16th and 31st
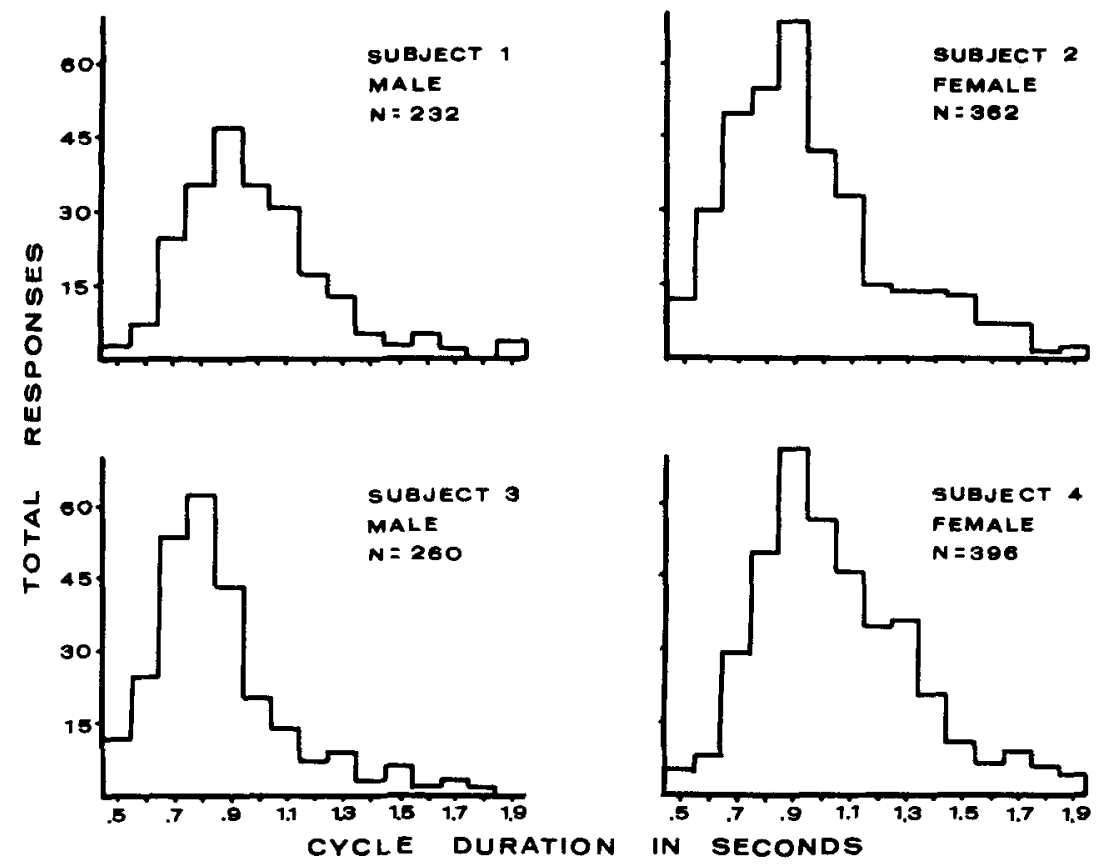

Fig. 5. Frequency distributions of chew cycle times for each $\mathbf{S}$.

chews in Fig. 3, lower frame.) Mфller (1966) made EMG recordings of the temporal muscles during chewing and swallowing and reported this difference in some detail. M $\phi$ ller found that electrical activity in the right anterior temporal muscle lasts about $300 \mathrm{msec}$ for each chew and about 1 sec for each swallow.

To ensure that recordings of chewing behavior are not confounded by other factors, Ss were asked to perform various behaviors, such as talking, smiling, nodding the head, and squinting of the eyes, while wearing the glasses. Intense squinting of the eyes and sharp jerking of the head to the side produced the largest voltage change, but in the most extreme case the voltage change was $1 / 10$ of that produced by eating peanuts. It appears then that the recordings are not greatly affected by other behaviors. The mode of modulation (FM) is relatively insensitive to variations in transmitter-to-receiver distances; thus, the $S$ may move around a rather wide range, approximately $10 \mathrm{ft}$, without affecting the data being collected.

\section{EXPLORATORY STUDY ON HUMAN CHEWING}

The chewing behavior of two male and two female introductory psychology students was recorded to demonstrate the capabilities of the apparatus and to generate some tentative ideas concerning individual differences in chewing behavior. The students volunteered for the study as part of a course requirement. Each was led to believe that it was a nutrition experiment exploring the relationship between blood-sugar level and reaction time.

The Ss were asked to eat caramel candy to increase their blood-sugar level, and they were told that the experiment measured body temperature changes from the temple and transmitted this information to receiving equipment in another room, thus explaining the need to wear the glasses and the necessity for taping a piece of material to the $S$ 's temple. Postexperimental questioning indicated that the Ss were completely unaware that their chewing was being recorded. The Ss were required to eat two pieces of caramel candy sometime during each of three 5-min rest periods. Each rest period was followed by a 2 -min reaction time test. All Ss were tested between 5 p.m. and 7 p.m., before eating their evening meal. Chewing movements were detected with the above-described telemetry system and they were recorded on a Texas Instruments, Model 327, hot pin Oscilloriter, running at $100 \mathrm{~mm} / \mathrm{sec}$ on the $1-\mathrm{V} / \mathrm{cm}$ range. A one-way mirror allowed the $\mathrm{E}$ to observe the S's chewing behavior at all times. The data recorded were similar to those shown in Fig. 3 (lower frame).

Cycle times were calculated by measuring the time interval between the beginning of one bite to the start of the next. Figure 5 shows a frequency distribution of these cycle times for each $\mathrm{S}$ in $0.1-\mathrm{sec}$ categories. Cycle times of $2 \mathrm{sec}$ and longer were considered pauses and not included in the distribution. The form of the distribution was similar for each $S$. 
Each was unimodal and relatively steep. The modal category was the same for each $S(0.9 \mathrm{sec})$, except No. 3 , whose mode was 0.8 sec.

The most salient difference between the Ss appeared to be in the total number of chews emitted. Both females emitted considerably more chews than the males, even though the same amount of foodstuff was consumed. This suggests that idividual differences in eating times may not be due to individuals chewing at different rates (i.e., chews/sec) but may be due to some people's emitting more chews at the same rate.

\section{SUMMARY}

The telemetry system described above provides a simple yet reliable method of detecting and recording human chewing behavior. It does not drastically restrict the $S$ 's movement or call attention to the behavior under study, as do the electromyographic and cinematographic methods. No special dental skills are required, as are necessary with tooth transmitters. The system has been used in several different exploratory studies, during which the chewing behavior of over 60 Ss, ranging in age from 7 to 72 , has been successfully recorded. Except for batteries, no component failures have been encountered.

\section{REFERENCES}

AHLGREN, J. Mechanism of mastication. Acta Odontologia Scandinavica, 1966 , 24, Supplement 44

BEYRON, $H$, Occlusal relations and mastication in Australian aborigines. Acta Odontologia Scandinavica, 1964, 22, 597

DUCKWORTH, J, E. \& SHIRLAW, D. W. Study of factors affecting feed intake and the eating behavior of cattle. Animal Behaviour, 1958, 6, 147-154.

FREEMAN, G. L. Dr. Hollingworth on chewing as a technique of relaxation. Psychological Review, 1940, 47, 491-493.

HOLLINGWORTH, H. L. Chewing as a technique of relaxation. Science, 1939 $90,385-387$.
JENKINS, G. N. The physiology of the mouth. (2nd ed.) Oxford: Blackwell, 1960.

KAVANAGH， D., \& ZANDER，H. A versatile recording system for studies of mastication. Medical Electronics Biological Engineering, 1965, 3, 291-300.

KAWAMURA, $Y$ Recent concepts of the physiology of mastication. In P. M. Staple (Ed.), Advances in oral biology. Vol. 1. New York: Academic Press, 1964. Pp. 77-109.

KLATSKY, M. Masticatory stresses and their relation to dental caries. Journal of Dental Research, 1942, 21, 389.

MOLLER, E. The chewing apparatus. Acta Physiologica Scandinavica, 1966, 69, Supplement 280.

ROEBER * F. W., GLICKMAN, I., \& PAMEIJER, J. H. N. An intraoral electronic system for the study of dental occlusion. Journal of Medical \& Biological Engineering, 1968, 6, 677-679.

RUGH, J. D. A telemetry system for recording mastication in small animals. Journal of Medical \& Biological Engineering, 1970, 8

WOLFF $\mathrm{S}$. Telemetry of psychophysiological variables. In $\mathrm{P}$. $\mathrm{H}$ Venables and I. Martin (Eds.), A manual of psychophysiological methods. New York: Wiley, 1967. 